\title{
Pharmacogenomics DNA Biomarkers in Colorectal Cancer: Current Update
}

\author{
Nurul-Syakima Ab Mutalib*, Najwa F. Md Yusof, Shafina-Nadiawati Abdul and \\ Rahman Jamal* \\ UKM Medical Molecular Biology Institute, UKM Medical Centre, Universiti Kebangsaan Malaysia, Kuala Lumpur, Malaysia
}

OPEN ACCESS

Edited by:

Moyra Smith,

University of California, Irvine

United States

Reviewed by:

Nelson Shu-Sang Yee,

Penn State Health Milton S. Hershey

Medical Center, United States

Antonio Rozzi,

Istituto Neurotraumatologico Italiano,

Italy

${ }^{*}$ Correspondence:

Nurul-Syakima Ab Mutalib

syakima@ppukm.ukm.edu.my

Rahman Jamal

rahmanj@ppukm.ukm.edu.my

Specialty section:

This article was submitted to

Cancer Molecular Targets and Therapeutics,

a section of the journal

Frontiers in Pharmacology

Received: 28 July 2017

Accepted: 29 September 2017

Published: 12 October 2017

Citation:

Ab Mutalib N-S, Md Yusof NF Abdul S-N and Jamal R (2017) Pharmacogenomics DNA Biomarkers in Colorectal Cancer: Current Update.

Front. Pharmacol. 8:736.

doi: 10.3389/fphar.2017.00736
Colorectal cancer (CRC) remains as one of the most common cause of worldwide cancer morbidity and mortality. Improvements in surgical modalities and adjuvant chemotherapy have increased the cure rates in early stage disease, but a significant portion of the patients will develop recurrence or advanced disease. The efficacy of chemotherapy of recurrence and advanced CRC has improved significantly over the last decade. Previously, the historical drug 5-fluorouracil was used as single chemotherapeutic agent. Now with the addition of other drugs such as capecitabine, irinotecan, oxaliplatin, bevacizumab, cetuximab, panitumumab, vemurafenib, and dabrafenib, the median survival of patients with advanced CRC has significantly improved from less than a year to the current standard of almost 2 years. However, the side effects of systemic therapy such as toxicity may cause fatal complications and have a major consequences on the patients' quality of life. Hence, there is an urgent need for key biomarkers which will enable the selection of optimal drug singly or in combination for an individual patient. The application of personalized therapy based on DNA testing could aid the clinicians in providing the most effective chemotherapy agents and dose modifications for each patient. Yet, some of the current findings are controversial and the evidences are conflicting. This review aims at summarizing the current state of knowledge about germline pharmacogenomics DNA variants that are currently used to guide therapeutic decisions and variants that have the potential to be clinically useful in the future. In addition, current updates on germline variants conferring treatment sensitivity, drug resistance to existing chemotherapy agents and variants affecting prognosis and survival will also be emphasized. Different alteration in the same gene might confer resistance or enhanced sensitivity; and while most of other published reviews generally stated only the gene name and codon location, we will specifically discuss the exact variants to offer more accurate information in this mini review.

Keywords: pharmacogenomics, precision medicine, colorectal cancer, DNA sequencing, gene variants, actionable target

\section{OVERVIEW OF SYSTEMIC CHEMOTHERAPY REGIMENS IN COLORECTAL CANCER}

The evolution of chemotherapy for colorectal cancer (CRC) was instigated with the development of 5-fluorouracil (5-FU) in 1957 (Heidelberger et al., 1957). Heidelberger et al. (1957) at the University of Wisconsin witnessed that tumor cells favored uracil for nucleic acid biosynthesis, and accurately hypothesized that a fluorouracil analog would inhibit tumor cell division by blocking 
the conversion of deoxyuridine monophosphate (dUMP) to deoxythymidine monophosphate (dTMP). The next landmark in CRC chemotherapy history was the discovery of 5-FU antitumor activity potentiation by leucovorin, which led to a large number of phase I and II clinical trials for metastatic CRC (mCRC) in the 1980s (Mini et al., 1990). Leucovorin is the folic acid in its reduced form and it is metabolized into tetrahydrofolate including 5,10- $\mathrm{CH}_{2}-\mathrm{FH}_{4}$ (Rustum, 1991). 5,10- $\mathrm{CH}_{2}-\mathrm{FH}_{4}$ is a cofactor that plays the role as a methyl donor for the dUMP conversion to dTMP by thymidylate synthase (TS) and is also important for interaction of FdUMP (one of the 5-FU active metabolites) with TS (Zaniboni, 1997). FdUMP, TS, and 5,10- $\mathrm{CH}_{2}-\mathrm{FH}_{4}$ form a covalent ternary complex whose stabilization leads to prolonged inhibition of TS activity and consequently, inhibition of DNA synthesis (Zaniboni, 1997). A meta-analysis of nine randomized clinical trials proved that leucovorin/5-FU combination increases the response rate from 11 to $23 \%$ compared to 5 -FU alone in mCRC (Piedbois et al., 1992). Despite being a backbone to CRC treatment, 5-FU possess a short half-life and constantly require infusions. Prolonged infusion was cumbersome for patients and can result in medical complications (Johnston and Kaye, 2001). Therefore an oral formulation of the drug is deemed necessary. In 2005, capecitabine was sanctioned by the Food and Drug Administration (FDA) as an oral prodrug of 5-FU to be used as monotherapy in the adjuvant setting for treating advanced stage CRC (Hirsch and Zafar, 2011).

The standard practice for 5-FU dosing is based on patient's body surface area (BSA); even though there is no significant correlation between the plasma clearance of 5-FU and BSA (Lee et al., 2016). An alternative pharmacokinetically guided (PKG) dosing strategy based on area under the concentration versus time curve (AUC) has remarkably improves the treatment response and lowered the risk of grade 3/4 toxicity for locally advanced CRC (Chen and Yee, 2016; Fang et al., 2016). However, further validation on this AUC-based PKG dosing focusing on prospective large-scale, randomized trials is still warranted (Chen and Yee, 2016). Tumor genetic makeup information, combined with AUC-based PKG dosing of 5-FU is foreseen to advance the development of personalized treatment in CRC patients further (Chen and Yee, 2016).

In the early 2000s, introduction of oxaliplatin and irinotecan as components of combination therapies for advanced disease marked another significant development in mCRC treatment (Gustavsson et al., 2015). Oxaliplatin, a platinum-containing agent, was discovered by Yoshinori Kidani in 1976 (Kidani et al., 1976, 1980) while irinotecan, a topoisomerase I inhibitor, was first discovered and synthesized by Yakult Honsha Ltd. in 1983 (Kunimoto et al., 1987). The effectiveness of irinotecan and oxaliplatin was first shown in patients that exhibited poor response to 5-FU/leucovorin, whereby 5-FU and leucovorin in combination with irinotecan (FOLFIRI) or oxaliplatin (FOLFOX) result in significantly increased response rates and improved survival compared to 5-FU/leucovorin alone (Douillard et al., 2000; MOSAIC study by André et al., 2004; NSABPC-07 study by Kuebler et al., 2007).

Another key development in the systemic management of mCRC was the advent of monoclonal antibodies targeting the vascular endothelial growth factor (VEGF) pathway or epidermal growth factor receptor (EGFR) (Cunningham et al., 2004; Hurwitz et al., 2004; Giantonio et al., 2007; Van Cutsem et al., 2007, 2009). Presently obtainable targeted agents active in mCRC include the anti-VEGF antibody (bevacizumab), the anti-EGFR antibodies (panitumumab and cetuximab), and the anti-VEGF agents (regorafenib and aflibercept). Due to these advancement in drug development, the median survival of CRC patients has significantly improved; yet, the side effects of systemic therapy such as toxicity, have become an important issue. Hence, there is an urgent need for biomarkers which could accurately predict each patient's response to each of the drug to enable optimal dosing or to provide alternative treatment. Table $\mathbf{1}$ summarizes the list of germline pharmacogenomics variants associated or potentially associated with adverse events following chemotherapy in CRC patients.

\section{DPYD}

The most commonly discussed marker of 5-FU toxicity is dihydropyrimidine dehydrogenase (DPD), an enzyme encoded by the DPYD, a large gene $(950 \mathrm{~kb})$ spanning 4,399 nucleotides in 23 coding exons on chromosome 1p22 (Wei et al., 1998). DPYD catalyzes 5-FU inactivation into dihydrofluorouracil, typically in the liver (Caudle et al., 2013) and deactivates more than 85\% of standard doses of 5-FU and capecitabine (Boige et al., 2016). Reduced or lack of DPD enzymatic activity affects approximately $5 \%$ of the overall population, subsequently extend the half-life of the drug, leading to excess accumulation of drug and toxicity (Lee et al., 2004). Besides, 3-5\% of the population has an incomplete DPD deficiency due to sequence alterations in DPYD gene, which possibly limits the ability of the liver to fully metabolize fluorouracil, thereby resulting in toxicity (Boige et al., 2016; Dhelens et al., 2016).

Clinical Pharmacogenetic Implementation Consortium (CPIC) has proposed genotype-based dosage recommendation for 5-FU according to the presence of DYPD allelic variants IVS14+1G $>$ A (*2A allele), I560S (*13 allele) and D949V (Offer et al., 2014). The CPIC proposes a substitute drug for homozygous patients and 50\% starting dose reduction for heterozygous patients. For the ease of understanding and to be currently relevant, variants will be now referred to by the amino acid change for which they encode.

The splice site variant IVS14+1G $>$ A is the most well-known single nucleotide polymorphism resulting in 5-FU toxicity. Located at the exon 14 intron border, this polymorphism leads to a splicing defect, causing skipping of the whole exon and produces a non-functional truncated protein (Van Kuilenburg et al., 1997). This splice variant is significantly associated with reduced 5-FU degradation rate (Gentile et al., 2016). It is strongly associated with a severe and life-threatening toxicity in treatment regimens which include capecitabine alone or in combination with others such as oxaliplatin, bevacizumab, cetuximab, 5-FU, or tegafur-uracil (Deenen et al., 2011, 2016; Meulendijks et al., 2015). The second well-known variant, I560S is remarkably infrequent in the general population, yet it has been constantly 
associated with reduced DPD activity (Offer et al., 2013a,b; Nie et al., 2017) and increased incidence of toxicity (Meulendijks et al., 2015; Dhelens et al., 2016). Clinical studies have also constantly proven the association between the third variant, D949V, and severe toxicity after chemotherapy that incorporated 5-FU. Patients with D949V necessitate significant capecitabine dose reduction due to severe toxicity (Deenen et al., 2011). Lee et al. (2014) also reported statistically significant associations between $\mathrm{D} 949 \mathrm{~V}$ and grade $\geq 3$ adverse events in patients treated with FOLFOX alone or combined with cetuximab. More recently, Boige et al. (2016) confirmed the significant impact of D949V in patients treated with FOLFOX4 with or without cetuximab. Collectively, these three alleles are the only clinically implemented variants to date (Whirl-Carrillo et al., 2012).

Even though many studies has reported the significant association between these three biomarkers with reduced DPD activity and 5-FU toxicity, its prediction power is restricted by low minor allele frequency across the general population (Cross et al., 2010). Furthermore, ethnicity seems to be playing a role with certain variants significantly associated with toxicity or reduced enzyme activity in one population but not observed in other population, most likely due to differences in allelic frequency. These findings demonstrate a serious

TABLE 1 | List of pharmacogenomics germline variants associated or potentially associated with toxicity or drug response in CRC patients.

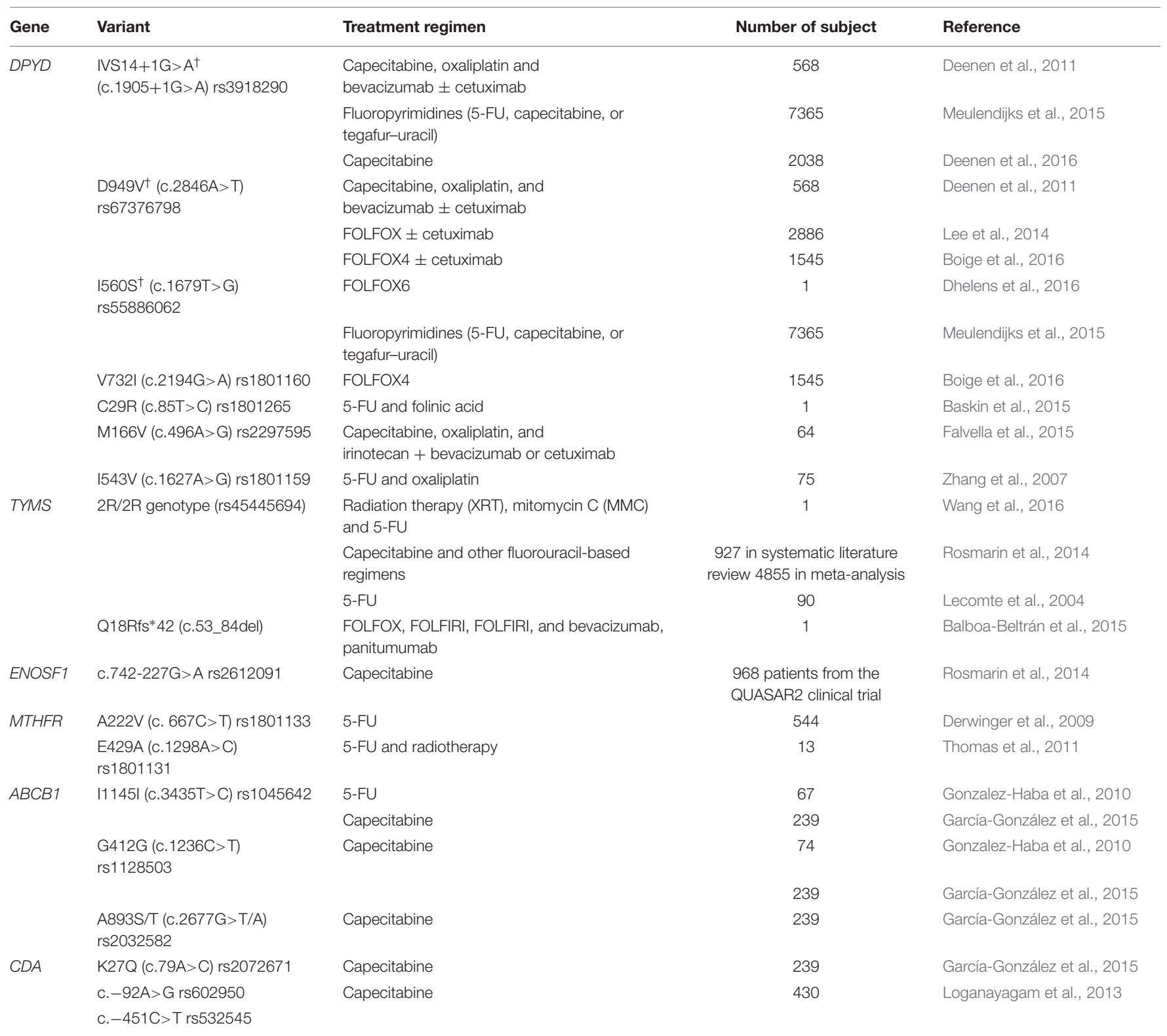

${ }^{\dagger}$ Clinically implemented. 5-FU, 5-fluorouracil; FOLFOX, fluorouracil, leucovorin, and oxaliplatin; FOLFIRI, folinic acid (leucovorin), fluorouracil and irinotecan; bFOL, bolus 5-FU, leucovorin and oxaliplatin. 
knowledge gap in pharmacogenetic biomarkers of 5-FU toxicity, which has been previously studied in populations of limited diversity.

In a substudy by PETACC- 8 randomized phase III clinical trial, genotyping in 1,545 patients revealed the association of D949V and also V732I with adverse events following FOLFOX4 treatment with or without cetuximab (Boige et al., 2016). The significant association of V732I was further replicated in an independent cohort of 339 mCRC patients receiving FOLFOX4 in the FFCD 2000-05 phase III trial (Ducreux et al., 2011). The PETACC- 8 is the only trial which demonstrated the association between V732I and fluorouracil-related adverse event so far.

There are many other DPYD variants reported but with weak evidence and conflicting data regarding the link between the variants with reduced 5-FU metabolism or increased 5-FU toxicity. For example, C29R (*9A) was identified in a DPD-deficient patient (Vreken et al., 1997a) and shown to be catalytically inactive (Vreken et al., 1997b); however, clinical studies fail to demonstrate its relationship with toxicity (Caudle et al., 2013; Offer et al., 2014). Nevertheless, there is one case report linking C29R with ocular toxicity in a CRC patient when treated with 5 -FU and folinic acid (Baskin et al., 2015). On the other hand, another study proposed that C29R may even has protective effect against 5-FU toxicity (Kleibl et al., 2009). Another variant, M166V, was revealed to be strongly linked with grade 3-4 toxicity in breast and gastroesophageal cancer patients, but not in CRC patients upon treated with a 5-FU-based therapy (Gross et al., 2008). Another three independent studies also unable to rectify a link between M166V and toxicity associated to 5-FU-based treatment (Amstutz et al., 2009; Deenen et al., 2011; Loganayagam et al., 2013). To date, the significant association between M166V and toxicity was only demonstrated by Falvella et al. (2015) who performed univariate and multivariate analysis involving 64 patients, in which the treatment regiments included capecitabine, oxaliplatin and irinotecan with bevacizumab or cetuximab. In another study involving 75 gastric and colon carcinoma patients, I543V (*5) leads to the decreased enzyme activity and significant toxicity toward 5-FU (Zhang et al., 2007); however, the association was not significant in PETACC-8 randomized phase III clinical trial (Boige et al., 2016).

The ability of NGS technology in enabling sequencing of the whole coding sequence has resulted in discovery of various novel $D P Y D$ variants whose functions and association with toxicity remain unclear. Nevertheless, several studies have linked the novel genotype to the respective phenotype characteristics. For example, the three clinically implemented variants (IVS14+1G $>$ A, I560S and D949V) were not detected in any of the 588 individuals of Somali or Kenyan ancestry living in central/southeast Minnesota (Elraiyah et al., 2017). However, via NGS, the authors identified several novel DPYD variants including I971Nfs which resulted in the complete lack of enzyme activity, and P86L, T793I, V941A, P1023S, A513V, and $\mathrm{P} 237 \mathrm{~L}$, in which all lead to significantly reduced DPD activity.

\section{TYMS AND ENOSF1}

Thymidylate synthase is considered to be a key intracellular target of fluoropyrimidines and is involved in dUMP conversion to dTMP, which is the exclusive source of thymidylate, a vital precursor for DNA synthesis (Heidelberger et al., 1957). Therefore, another candidate gene to predict 5-FU toxicity is the TS gene (TYMS), which is strongly inhibited by 5 -FU (Shahrokni et al., 2009). This gene is located on the chromosome 18 at p11.32 which covers seven exons (Jakobsen et al., 2005). The TYMS promoter encompasses of a 28 -bp sequence in the $5^{\prime}$-untranslated enhanced region $\left(5^{\prime}\right.$-UTR) and presented as a double-tandem repeat (2R) or a triple-tandem repeat (3R) (Huang et al., 2016).

Triple-tandem repeat $(3 \mathrm{R})$ and double-tandem repeat (2R) polymorphisms are associated with TYMS expression, 5-FU response and toxicity in a different manner. The $2 \mathrm{R} / 2 \mathrm{R}$ genotype exhibits significantly lower TS mRNA levels compared to 3R/3R or 2R/3R (Pullarkat et al., 2001). Homozygous 2R/2R is significantly associated with increased susceptibility to 5 -FU toxicity and drug sensitivity (Lecomte et al., 2004; Shahrokni et al., 2009). This fact is further strengthen by a meta-analysis in 4,855 patients by Rosmarin et al. (2014) which involved QUASAR2 and 16 other published studies. More recently, a case report on a CRC patient with homozygous $2 \mathrm{R} / 2 \mathrm{R}$ receiving 5 -FU therapy, mitomycin-C and adjuvant radiation who develop severe gastrointestinal toxicities and pancytopenia associated with 5-FU (Wang et al., 2016).

On the other hand, 3R polymorphism confers different effect compared to 2R. Pullarkat et al. (2001) suggest that $3 \mathrm{R}$ allele is responsible for 3.6 times higher TYMS in patients with mCRC compared to patients who were carrying $2 \mathrm{R}$ variant. Patients with the $3 \mathrm{R} / 3 \mathrm{R}$ genotype had significantly less severe side effect and toxicity with 5 -FU-based chemotherapy when compared to the $2 \mathrm{R} / 2 \mathrm{R}$ genotype (Pullarkat et al., 2001). However, 3R/3R polymorphism will render the treatment ineffective, which subsequently lead to poor prognosis. Due to their distinct consequences, these two variations pose a dilemma in 5-FU-based cancer therapy; whereby high expression by $3 \mathrm{R} / 3 \mathrm{R}$ leads to reduced drug sensitivity, whereas low expression by $2 \mathrm{R} / 2 \mathrm{R}$ leads to the drug toxicity that may cause cessation of the therapy.

TYMS tandem repeat polymorphisms are the most studied alteration in this gene to date. However, novel mutation in coding regions have also begun to be revealed. For instance, Balboa-Beltrán et al. (2015) reported the first discovery of heterozygous germline stop codon mutation Q18Rfs*42 in a mCRC patient which cause loss of function of one of the TYMS alleles, resulting in a truncated protein. Despite of a long survival (more than eight years after diagnosis), the patient developed severe toxicity under 5-FU-based therapy. Due to the absence of DYPD IVS14+1G>A, D949V, I560S and several other markers in relation to toxicity, the authors hypothesize that TYMS Q18Rfs*42 could be the cause of severe toxicities suffered by the patient.

ENOSF1 is a poorly characterized gene located adjacent to TYMS, and is hypothesized to code a protein and also an 
antisense transcript to TYMS, thus regulating its mRNA and/or protein expression (Dolnick et al., 2003). Rosmarin et al.'s (2014) identified a significant association between capecitabine toxicity and ENOSF1 c.742-227G $>$ A. The authors further concluded that the presence of this intronic variant was the cause of 5-FU toxicity reported in patients with functional TYMS but harboring 50 VNTR and 30 UTR polymorphisms (Rosmarin et al., 2014).

\section{MTHFR}

Methylenetetrahydrofolate reductase is encoded by MTHFR gene and could also be an important predictor of response toward 5-FU. Methylenetetrahydrofolate reductase catalyzes the change of 5-10-methylenetetrahydrofolate, which is essential to the DNA synthesis, to 5-methyltetrahydrofolate by acting as a cofactor in conversion of dUMP to dTMP by TS (Jakobsen et al., 2005). In addition, this enzyme maintains the stability of the binding of 5-fluorodeoxyuridine monophosphate to TS, subsequently results in sustained inhibition of the enzyme.

Several single nucleotide polymorphisms (SNPs) are shown to influence the activity of MTHFR, and the variant with the significant association with 5-FU response in CRC is MTHFR A222V. It results in a substantial lower activity and thermolability of the enzyme in heterozygotes and homozygotes variant compared to the wild-type homozygotes (Sohn et al., 2004). Subsequently, a buildup of 5-10-methylenetetrahydrofolate is observed, and increased 5-FU sensitivity is anticipated. In vitro studies have identified the MTHFR A222V as a vital predictor of 5-FU response, where it changes intracellular folate distribution, accelerates cellular growth rate, increases TS activity and chemosensitivity of CRC cells to 5-FU (Sohn et al., 2004). A study by Jakobsen et al. (2005) proved the significant association of MTHFR A222V polymorphism with increased 5-FU response. The association was further demonstrated by Derwinger et al. (2009) who concluded that MTHFR A222V not only affect the 5-FU sensitivity, but also increased the risk of unwanted side-effects and subsequently affecting survival in stage III and stage IV CRC. Etienne-Grimaldi et al. (2010) showed that even though there was a significant association between MTHFR $\mathrm{A} 222 \mathrm{~V}$ and increase 5-FU response, this variant is not a toxicity predictor. On the other hand, Thomas et al. (2011) reported that MTHFR E429A and MTHFR diplotypes (for A222V and E429A) were significantly connected to toxicity when only 5FU was used. In contrast Etienne-Grimaldi et al. (2010) showed that there was no association between these biomarkers and drug response.

Another study using capecitabine monotherapy and capecitabine-irinotecan in mCRC reported conflicting results (van Huis-Tanja et al., 2013). The authors showed that MTHFR E429A and A222V genotypes were not associated with efficacy or toxicity in MCRC patients. Comparisons among different genotypes of MTHFR 1298 locus revealed that CC homozygotes exhibited greater incidence of grade 3-4 diarrhea compared with AC or AA individuals but only at borderline significance. Therefore, it remains controversial whether MTHFR polymorphisms can possibly predict toxicity or 5-FU response in patients treated with fluoropyrimidines.

\section{ABCB1}

The $A B C B 1$ gene, located on chromosome 7 (Schrickx and Fink-Gremmels, 2008) belongs to drug transporter gene family. T carriers of $A B C B 1$ I1145I treated with 5-FU had a significantly higher risk of diarrhea (García-González et al., 2015), while the same genotype conferred a lower risk of hand-and-foot syndrome when treated with capecitabine (Gonzalez-Haba et al., 2010), despite these two drugs belong in the same class (fluoropyrimidines). The association between $A B C B 1^{*} 1$ haplotype [1236C, 2677G (893Ala), and $3435 \mathrm{C}$ ] with severe overall toxicity was significant in 239 capecitabine-treated CRC patients (García-González et al., 2015).

\section{$C D A$}

The CDA gene, located on chromosome 1 (Saccone et al., 1994), encodes an enzyme in the pyrimidine salvage pathway and is important for metabolism of antitumor cytosine nucleoside analogs, leading to 5-FU activation (Lou et al., 2016). Despite scarce evidence, the relationship between CDA promoter variants and 5-FU toxicity in $\mathrm{CRC}$ has been demonstrated. $C D A$ c. $-92 \mathrm{~A}>\mathrm{G}$ and c. $-451 \mathrm{C}>\mathrm{T}$ variants were significantly associated with grade 2-4 diarrhea (Loganayagam et al., 2013), while exonic K27Q AA is significantly associated with $\geq 3$ overall toxicity (García-González et al., 2015).

\section{BED-TO-BEDSIDE CHALLENGES, FUTURE RECOMMENDATION, AND CONCLUSIONS}

In summary, we have reviewed the current information of potential pharmacogenetics gene variants that could help the oncologists in making therapeutic decisions. The clinical utility of DPYD IVS14+1G > A, D949V, and I560S have been demonstrated in a number of large prospective/retrospective studies and clinical trials; however, the discrepancies of other DPYD variants among multistudies could be due to small cohorts that include diverse stages and treatment regimens. Lesser evidence is available for TYMS, ENOSF1, MTHFR, $A B C B 1$, and $C D A$; nevertheless, at least one study which includes hundreds of CRC patients has reported the significant association among the altered genes with adverse events and this information should not be disregarded. Nonetheless, the role of variants in TYMS, ENOSF1, MTHFR, ABCB1, and $C D A$ genes still warrant for additional validation in larger cohort.

The clinical application of precision medicine is hindered by several disagreements such as the unwillingness of clinicians, ministry of health, and insurance organizations to adopt this 
into clinical practice. Unless we overcome these major hurdles, precision medicine will have a long way to go. While the former president of the United States, Barack Obama, had declared the Precision Medicine initiative in 2015, other countries have yet to embark on a similar initiative especially so in the developing nations, hence more effort still need to be done to increase awareness of precision medicine.

The role of ethnic differences in drug response or toxicity is accepted as a crucial factor responsible for interindividual variation in anticancer drug sensitivity (O'Donnell and Dolan, 2009; Mohelnikova-Duchonova et al., 2014). A toxicity biomarkers in Caucasian might differ from Asian or African descendant patients. Therefore, identification of ethnic-specific biomarkers for drug response is imperative. Information compiled in this review could be applied in designing a targeted gene panel for personalized medicine in CRC. Genotyping of these genes in advance before chemotherapy could help to lessen the adverse reactions in CRC patients.

\section{REFERENCES}

Amstutz, U., Farese, S., Aebi, S., and Largiadèr, C. R. (2009). Dihydropyrimidine dehydrogenase gene variation and severe 5-fluorouracil toxicity: a haplotype assessment. Pharmacogenomics 10, 931-944. doi: 10.2217/pgs.09.28

André, T., Boni, C., Mounedji-Boudiaf, L., Navarro, M., Tabernero, J., Hickish, T., et al. (2004). Oxaliplatin, fluorouracil, and leucovorin as adjuvant treatment for colon cancer. N. Engl. J. Med. 350, 2343-2351. doi: 10.1056/NEJMoa032709

Balboa-Beltrán, E., Duran, G., Lamas, M. J., Carracedo, A., and Barros, F. (2015). Long survival and severe toxicity under 5-fluorouracil-based therapy in a patient with colorectal cancer who harbors a germline codon-stop mutation in TYMS. Mayo Clin. Proc. 90, 1298-1303. doi: 10.1016/j.mayocp.2015.05.005

Baskin, Y., Amirfallah, A., Unal, O. U., Calibasi, G., and Oztop, I. (2015). Dihydropyrimidine dehydrogenase $85 \mathrm{~T}>\mathrm{C}$ mutation is associated with ocular toxicity of 5-fluorouracil: a case report. Am. J. Ther. 22, e36-e39. doi: 10.1097/ MJT.0b013e31829e8516

Boige, V., Vincent, M., Alexandre, P., Tejpar, S., Landolfi, S., Le Malicot, K., et al. (2016). DPYD genotyping to predict adverse events following treatment with flourouracil-based adjuvant chemotherapy in patients with stage III colon cancer: a secondary analysis of the petacc- 8 randomized clinical trial. JAMA Oncol. 2, 655-662. doi: 10.1001/jamaoncol.2015.5392

Caudle, K. E., Thorn, C. F., Klein, T. E., Swen, J. J., McLeod, H. L., Diasio, R. B., et al. (2013). Clinical Pharmacogenetics Implementation Consortium guidelines for dihydropyrimidine dehydrogenase genotype and fluoropyrimidine dosing. Clin. Pharmacol. Ther. 94, 640-645. doi: 10.1038/clpt.2013.172

Chen, Y., and Yee, N. S. (2016). Pharmacokinetics-guided dosing of 5-fluorouracil for precision cancer treatment: a focus on colorectal carcinoma. Appl. Clin. Res. 3, 159-163. doi: 10.2174/2213476X03666160902124427

Cross, D. S., Ivacic, L. C., Stefanski, E. L., and McCarty, C. A. (2010). Population based allele frequencies of disease associated polymorphisms in the Personalized Medicine Research Project. BMC Genet. 11:51. doi: 10.1186/14712156-11-51

Cunningham, D., Humblet, Y., Siena, S., Khayat, D., Bleiberg, H., Santoro, A., et al. (2004). Cetuximab monotherapy and cetuximab plus irinotecan in irinotecanrefractory metastatic colorectal cancer. N. Engl. J. Med. 351, 337-345. doi: 10.1056/NEJMoa033025

Deenen, M. J., Meulendijks, D., Cats, A., Sechterberger, M. K., Severens, J. L., Boot, H., et al. (2016). Upfront genotyping of DPYD*2A to individualize fluoropyrimidine therapy: a safety and cost analysis. J. Clin. Oncol. 34, 227-234. doi: 10.1200/JCO.2015.63.1325

Deenen, M. J., Tol, J., Burylo, A. M., Doodeman, V. D., de Boer, A., Vincent, A., et al. (2011). Relationship between single nucleotide polymorphisms and

\section{AUTHOR CONTRIBUTIONS}

N-SA, NFM, and S-NA drafted this manuscript. N-SA and RJ were responsible for idea conception, critical evaluation, and manuscript review.

\section{FUNDING}

This review was funded by Long Research Grant Scheme (LRGS/2014/UKM-UKM/K/01) and Higher Institutions Centre of Excellence (HICoE) grant (10-64-01-005) from Ministry of Higher Education Malaysia.

\section{ACKNOWLEDGMENTS}

The authors acknowledge the contributions of Ministry of Higher Education and Universiti Kebangsaan Malaysia in supporting the works.

haplotypes in DPYD and toxicity and efficacy of capecitabine in advanced colorectal cancer. Clin. Cancer Res. 17, 3455-3468. doi: 10.1158/1078-0432. CCR-10-2209

Derwinger, K., Wettergren, Y., Odin, E., Carlsson, G., and Gustavsson, B. (2009). A study of the MTHFR gene polymorphism C677T in colorectal cancer. Clin. Colorectal Cancer 8, 43-48. doi: 10.3816/CCC.2009.n.007

Dhelens, C., Bonadona, A., Thomas, F., Chapuis, C., Potton, L., Marsili, S., et al. (2016). Lethal 5-fluorouracil toxicity in a colorectal patient with severe dihydropyrimidine dehydrogenase (DPD) deficiency. Int. J. Colorectal Dis. 31, 699-701. doi: 10.1007/s00384-015-2191-0

Dolnick, B. J., Angelino, N. J., Dolnick, R., and Sufrin, J. R. (2003). A novel function for the rTS gene. Cancer Biol. Ther. 2, 364-369. doi: 10.4161/cbt.2.4.424

Douillard, J., Cunningham, D., Roth, A. D., Navarro, M., James, R. D., Karasek, P., et al. (2000). Irinotecan combined with fluorouracil compared with fluorouracil alone as first-line treatment for metastatic colorectal cancer: a multicentre randomised trial. Lancet 355, 1041-1047. doi: 10.1016/S0140-6736(00) 02034-1

Ducreux, M., Malka, D., Mendiboure, J., Etienne, P. L., Texereau, P., Auby, D., et al. (2011). Sequential versus combination chemotherapy for the treatment of advanced colorectal cancer (FFCD 2000-05): an open-label, randomised, phase 3 trial. Lancet Oncol. 12, 1032-1044. doi: 10.1016/S1470-2045(11)70199-1

Elraiyah, T., Jerde, C. R., Shrestha, S., Wu, R., Nie, Q., Giama, N. H., et al. (2017). Novel deleterious dihydropyrimidine dehydrogenase variants may contribute to 5-fluorouracil sensitivity in an East African population. Clin. Pharmacol. Ther. 101, 382-390. doi: 10.1002/cpt.531

Etienne-Grimaldi, M. C., Milano, G., Maindrault-Goebel, F., Chibaudel, B., Formento, J. L., Francoual, M., et al. (2010). Methylenetetrahydrofolate reductase (MTHFR) gene polymorphisms and FOLFOX response in colorectal cancer patients. Br. J. Clin. Pharmacol. 69, 58-66. doi: 10.1111/j.1365-2125. 2009.03556.x

Falvella, F. S., Cheli, S., Martinetti, A., Mazzali, C., Iacovelli, R., Maggi, C., et al. (2015). DPD and UGT1A1 deficiency in colorectal cancer patients receiving triplet chemotherapy with fluoropyrimidines, oxaliplatin and irinotecan. Br. J. Clin. Pharmacol. 80, 581-588. doi: 10.1111/bcp.12631

Fang, L., Xin, W., Ding, H., Zhang, Y., Zhong, L., Luo, H., et al. (2016). Pharmacokinetically guided algorithm of 5-fluorouracil dosing, a reliable strategy of precision chemotherapy for solid tumors: a meta-analysis. Sci. Rep. 6:25913. doi: 10.1038/srep25913

García-González, X., Cortejoso, L., García, M. I., García-Alfonso, P., Robles, L., Grávalos, C., et al. (2015). Variants in CDA and ABCB1 are predictors of capecitabine-related adverse reactions in colorectal cancer. Oncotarget 6, 6422-6430. doi: 10.18632/oncotarget.3289 
Gentile, G., Botticelli, A., Lionetto, L., Mazzuca, F., Simmaco, M., Marchetti, P., et al. (2016). Genotype-phenotype correlations in 5-fluorouracil metabolism: a candidate DPYD haplotype to improve toxicity prediction. Pharmacogenomics J. 16, 320-325. doi: 10.1038/tpj.2015.56

Giantonio, B. J., Catalano, P. J., Meropol, N. J., O’Dwyer, P. J., Mitchell, E. P., Alberts, S. R., et al. (2007). Bevacizumab in combination with oxaliplatin, fluorouracil, and leucovorin (FOLFOX4) for previously treated metastatic colorectal cancer: results from the Eastern Cooperative Oncology Group Study E3200. J. Clin. Oncol. 25, 1539-1544. doi: 10.1200/JCO.2006.09. 6305

Gonzalez-Haba, E., García, M. I., Cortejoso, L., López-Lillo, C., Barrueco, N., García-Alfonso, P., et al. (2010). ABCB1 gene polymorphisms are associated with adverse reactions in fluoropyrimidine-treated colorectal cancer patients. Pharmacogenomics 11, 1715-1723. doi: 10.2217/pgs.10.159

Gross, E., Busse, B., Riemenschneider, M., Neubauer, S., Seck, K., Klein, H. G., et al. (2008). Strong association of a common dihydropyrimidine dehydrogenase gene polymorphism with fluoropyrimidine-related toxicity in cancer patients. PLOS ONE 3:e4003. doi: 10.1371/journal.pone.0004003

Gustavsson, B., Carlsson, G., Machover, D., Petrelli, N., Roth, A., Schmoll, H. J., et al. (2015). A review of the evolution of systemic chemotherapy in the management of colorectal cancer. Clin. Colorectal Cancer 14, 1-10. doi: 10.1016/j.clcc.2014.11.002

Heidelberger, C., Chaudhuri, N. K., Danneberg, P., Mooren, D., Griesbach, L., Duschinsky, R., et al. (1957). Fluorinated pyrimidines, a new class of tumourinhibitory compounds. Nature 179, 663-666. doi: 10.1038/179663a0

Hirsch, B. R., and Zafar, S. Y. (2011). Capecitabine in the management of colorectal cancer. Cancer Manag. Res. 3, 79-89. doi: 10.2147/CMR.S11250

Huang, K., Shen, Y., Zhang, F., Wang, S., and Wei, X. (2016). Evaluation of effects of thymidylate synthase and excision repair cross-complementing 1 polymorphisms on chemotherapy outcome in patients with gastrointestinal tumors using peripheral venous blood. Oncol. Lett. 11, 3477-3482. doi: 10.3892/ ol.2016.4423

Hurwitz, H., Fehrenbacher, L., Novotny, W., Cartwright, T., Hainsworth, J., Heim, W., et al. (2004). Bevacizumab plus irinotecan, fluorouracil, and leucovorin for metastatic colorectal cancer. N. Engl. J. Med. 350, 2335-2342. doi: 10.1056/NEJMoa032691

Jakobsen, A., Nielsen, N. J., Gyldenkerne, N., and Lindeberg, J. (2005). Thymidylate synthase and methylenetetrahydrofolate reductase gene polymorphism in normal tissue as predictors of fluorouracil sensitivity. J. Clin. Oncol. 23, 1365-1369. doi: 10.1200/JCO.2005.06.219

Johnston, P. G., and Kaye, S. (2001). Capecitabine: a novel agent for the treatment of solid tumors. Anticancer Drugs 12, 639-646. doi: 10.1097/00001813200109000-00001

Kidani, Y., Inagaki, K., and Tsukagoshi, S. (1976). Examination of antitumor activities of platinum complexes of 1,2-diaminocyclohexane isomers and their related complexes. Jpn. J. Cancer Res. 67, 921-922. doi: 10.20772/cancersci1959. 67.6_921

Kidani, Y., Noji, M., and Tashiro, T. (1980). Antitumor activity of platinum (II) complexes of 1, 2-diaminocyclohexane isomers. Jpn. J. Cancer Res. 71, 637-643. doi: 10.20772/cancersci1959.71.5_637

Kleibl, Z., Fidlerova, J., Kleiblova, P., Kormunda, S., Bilek, M., Bouskova, K., et al. (2009). Influence of dihydropyrimidine dehydrogenase gene (DPYD) coding sequence variants on the development of fluoropyrimidine-related toxicity in patients with high-grade toxicity and patients with excellent tolerance of fluoropyrimidine-based chemotherapy. Neoplasma 56, 303-316. doi: 10.4149/ neo_2009_04_303

Kuebler, J. P., Wieand, H. S., O’Connell, M. J., Smith, R. E., Colangelo, L. H., Yothers, G., et al. (2007). Oxaliplatin combined with weekly bolus fluorouracil and leucovorin as surgical adjuvant chemotherapy for stage II and III colon cancer: results from NSABP C-07. J. Clin. Oncol. 25, 2198-2204. doi: 10.1200/ JCO.2006.08.2974

Kunimoto, T., Nitta, K., Tanaka, T., Uehara, N., Baba, H., Takeuchi, M., et al. (1987). Antitumor activity of 7-ethyl-10-[4-(1-piperidino)-1piperidino]carbonyloxy-camptothec in, a novel water-soluble derivative of camptothecin, against murine tumors. Cancer Res. 47, 5944-5947.

Lecomte, T., Ferraz, J. M., Zinzindohoué, F., Loriot, M. A., Tregouet, D. A., Landi, B., et al. (2004). Thymidylate synthase gene polymorphism predicts toxicity in colorectal cancer patients receiving 5-fluorouracil-based chemotherapy. Clin. Cancer Res. 10, 5880-5888. doi: 10.1158/1078-0432.CCR04-0169

Lee, A., Ezzeldin, H., Fourie, J., and Diasio, R. (2004). Dihydropyrimidine dehydrogenase deficiency: impact of pharmacogenetics on 5-fluorouracil therapy. Clin. Adv. Hematol. Oncol. 2, 527-532.

Lee, A. M., Shi, Q., Pavey, E., Alberts, S. R., Sargent, D. J., Sinicrope, F. A., et al. (2014). DPYD variants as predictors of 5-fluorouracil toxicity in adjuvant colon cancer treatment (NCCTG N0147). J. Natl. Cancer Inst. 106:dju298. doi: $10.1093 /$ jnci/dju298

Lee, J. J., Beumer, J. H., and Chu, E. (2016). Therapeutic drug monitoring of 5fluorouracil. Cancer Chemother. Pharmacol. 78, 447-464. doi: 10.1007/s00280016-3054-2

Loganayagam, A., Hernandez, M. A., Corrigan, A., Fairbanks, L., Lewis, C. M., Harper, P., et al. (2013). Pharmacogenetic variants in the DPYD, TYMS, CDA and MTHFR genes are clinically significant predictors of fluoropyrimidine toxicity. Br. J. Cancer 108, 2505-2515. doi: 10.1038/bjc.2013.262

Lou, Y., Wang, Q., Zheng, J., Hu, H., Liu, L., Hong, D., et al. (2016). Possible pathways of capecitabine-induced hand-foot syndrome. Chem. Res. Toxicol. 29, 1591-1601. doi: 10.1021/acs.chemrestox.6b00215

Meulendijks, D., Henricks, L. M., Sonke, G. S., Deenen, M. J., Froehlich, T. K., Amstutz, U., et al. (2015). Clinical relevance of DPYD variants c.1679T $>\mathrm{G}$, c. $1236 \mathrm{G}>\mathrm{A} / \mathrm{HapB} 3$, and c. $1601 \mathrm{G}>\mathrm{A}$ as predictors of severe fluoropyrimidineassociated toxicity: a systematic review and meta-analysis of individual patient data. Lancet Oncol. 16, 1639-1650. doi: 10.1016/S1470-2045(15)00286-7

Mini, E., Trave, F., Rustum, Y. M., and Bertino, J. R. (1990). Enhancement of the antitumor effects of 5-fluorouracil by folinic acid. Pharmacol. Ther. 47, 1-19. doi: 10.1016/0163-7258(90)90042-Z

Mohelnikova-Duchonova, B., Melichar, B., and Soucek, P. (2014). FOLFOX/FOLFIRI pharmacogenetics: the call for a personalized approach in colorectal cancer therapy. World J. Gastroenterol. 20, 10316-10330. doi: 10.3748/wjg.v20.i30.10316

Nie, Q., Shrestha, S., Tapper, E. E., Trogstad-Isaacson, C. S., Bouchonville, K. J., Lee, A. M., et al. (2017). Quantitative contribution of rs75017182 to dihydropyrimidine dehydrogenase mRNA splicing and enzyme activity. Clin. Pharmacol. Ther. 102, 662-670. doi: 10.1002/cpt.685

O’Donnell, P. H., and Dolan, M. E. (2009). Cancer pharmacoethnicity: ethnic differences in susceptibility to the effects of chemotherapy. Clin. Cancer Res. 15, 4806-4814. doi: 10.1158/1078-0432.CCR-09-0344

Offer, S. M., Fossum, C. C., Wegner, N. J., Stuflesser, A. J., Butterfield, G. L., and Diasio, R. B. (2014). Comparative functional analysis of DPYD variants of potential clinical relevance to dihydropyrimidine dehydrogenase activity. Cancer Res. 74, 2545-2554. doi: 10.1158/0008-5472.CAN-13-2482

Offer, S. M., Lee, A. M., Mattison, L. K., Fossum, C., Wegner, N. J., and Diasio, R. B. (2013a). A DPYD variant (Y186C) in individuals of African ancestry is associated with reduced DPD enzyme activity. Clin. Pharmacol. Ther. 94, 158-166. doi: 10.1038/clpt.2013.69

Offer, S. M., Wegner, N. J., Fossum, C., Wang, K., and Diasio, R. B. (2013b). Phenotypic profiling of DPYD variations relevant to 5-fluorouracil sensitivity using real-time cellular analysis and in vitro measurement of enzyme activity. Cancer Res. 73, 1958-1968. doi: 10.1158/0008-5472.CAN-12-3858

Piedbois, P., Buyse, M., Rustum, Y., Machover, D., Erlichman, C., Carlson, R. W., et al. (1992). Modulation of fluorouracil by leucovorin in patients with advanced colorectal cancer: evidence in terms of response rate by the advanced colorectal cancer meta-analysis project. J. Clin. Oncol. 10, 896-903. doi: 10.1200/JCO. 1992.10.6.896

Pullarkat, S. T., Stoehlmacher, J., Ghaderi, V., Xiong, Y. P., Ingles, S. A., Sherrod, A., et al. (2001). Thymidylate synthase gene polymorphism determines response and toxicity of 5-FU chemotherapy. Pharmacogenomics J. 1, 65-70. doi: $10.1038 /$ sj.tpj. 6500012

Rosmarin, D., Palles, C., Church, D., Domingo, E., Jones, A., Johnstone, E., et al. (2014). Genetic markers of toxicity from capecitabine and other fluorouracilbased regimens: investigation in the QUASAR2 study, systematic review, and meta-analysis. J. Clin. Oncol. 32, 1031-1039. doi: 10.1200/JCO.2013.51.1857

Rustum, Y. M. (1991). Modulation of fluoropyrimidines by leucovorin: rational and status. J. Surg. Oncol. 48, 116-123. doi: 10.1002/jso.2930480525

Saccone, S., Andreozzi, L., and Della Valle, G. (1994). Assignment of the human cytidine deaminase (CDA) gene to chromosome 1 band p35-p36.2. Genomics 22, 661-662. doi: 10.1006/geno.1994.1448 
Schrickx, J. A., and Fink-Gremmels, J. (2008). Implications of ABC transporters on the disposition of typical veterinary medicinal products. Eur. J. Pharmacol. 585, 510-519. doi: 10.1016/j.ejphar.2008.03.014

Shahrokni, A., Rajebi, M. R., and Saif, M. W. (2009). Toxicity and efficacy of 5 fluorouracil and capecitabine in a patient with TYMS gene polymorphism: a challenge or a dilemma? Clin. Colorectal Cancer 8, 231-234. doi: 10.3816/CCC. 2009.n.039

Sohn, K. J., Croxford, R., Yates, Z., Lucock, M., and Kim, Y. I. (2004). Effect of the methylenetetrahydrofolate reductase C677T polymorphism on chemosensitivity of colon and breast cancer cells to 5-fluorouracil and methotrexate. J. Natl. Cancer Inst. 96, 134-144. doi: 10.1093/jnci/djh015

Thomas, F., Motsinger-Reif, A. A., Hoskins, J. M., Dvorak, A., Roy, S., Alyasiri, A., et al. (2011). Methylenetetrahydrofolate reductase genetic polymorphisms and toxicity to 5-FU-based chemoradiation in rectal cancer. Br. J. Cancer. 105, 1654-1662. doi: 10.1038/bjc.2011.442

Van Cutsem, E., Köhne, C. H., Hitre, E., Zaluski, J., Chang Chien, C. R., Makhson, A., et al. (2009). Cetuximab and chemotherapy as initial treatment for metastatic colorectal cancer. N. Engl. J. Med. 360, 1408-1417. doi: 10.1056/ NEJMoa0805019

Van Cutsem, E., Peeters, M., Siena, S., Humblet, Y., Hendlisz, A., Neyns, B., et al. (2007). Open-label phase III trial of panitumumab plus best supportive care compared with best supportive care alone in patients with chemotherapyrefractory metastatic colorectal cancer. J. Clin. Oncol. 25, 1658-1664. doi: 10.1200/JCO.2006.08.1620

van Huis-Tanja, L. H., Gelderblom, H., Punt, C. J., and Guchelaar, H. J. (2013). MTHFR polymorphisms and capecitabine-induced toxicity in patients with metastatic colorectal cancer. Pharmacogenet. Genomics 23, 208-218. doi: 10.1097/FPC.0b013e32835ee8e1

Van Kuilenburg, A. B., Vreken, P., Beex, L. V., Meinsma, R., Van Lenthe, H., De Abreu, R. A., et al. (1997). Heterozygosity for a point mutation in an invariant splice donor site of dihydropyrimidine dehydrogenase and severe 5-fluorouracil related toxicity. Eur. J. Cancer 33, 2258-2264. doi: 10.1016/S0959-8049(97) 00261-X

Vreken, P., Van Kuilenburg, A. B., Meinsma, R., and Van Gennip, A. H. (1997a). Identification of novel point mutations in the dihydropyrimidine dehydrogenase gene. J. Inherit. Metab. Dis. 20, 335-338. doi: 10.1023/A: 1005357307122

Vreken, P., Van Kuilenburg, A. B., Meinsma, R., and Van Gennip, A. H. (1997b). Dihydropyrimidine dehydrogenase (DPD) deficiency: identification and expression of missense mutations C29R, R886H and R235W. Hum. Genet. 101, 333-338. doi: 10.1007/s004390050637

Wang, B., Walsh, S. J., and Saif, M. W. (2016). Pancytopenia and severe gastrointestinal toxicities associated with 5-fluorouracil in a patient with thymidylate synthase (TYMS) polymorphism. Cureus 8:e798. doi: 10.7759/ cureus.798

Wei, X., Elizondo, G., Sapone, A., McLeod, H. L., Raunio, H., FernandezSalguero, P., et al. (1998). Characterization of the human dihydropyrimidine dehydrogenase gene. Genomics 51, 391-400. doi: 10.1006/geno.1998. 5379

Whirl-Carrillo, M., McDonagh, E. M., Hebert, J. M., Gong, L., Sangkuhl, K., Thorn, C. F., et al. (2012). Pharmacogenomics knowledge for personalized medicine. Clin. Pharmacol. Ther. 92, 414-417. doi: 10.1038/clpt.2012.96

Zaniboni, A. (1997). Adjuvant chemotherapy in colorectal cancer with highdose leucovorin, and fluorouracil: impact on disease-free survival, and overall survival. J. Clin. Oncol. 15, 2432-2441. doi: 10.1200/JCO.1997.15. 6.2432

Zhang, H., Li, Y. M., Zhang, H., and Jin, X. (2007). DPYD* 5 gene mutation contributes to the reduced DPYD enzyme activity and chemotherapeutic toxicity of 5-FU. Med. Oncol. 24, 251-258. doi: 10.1007/BF02698048

Conflict of Interest Statement: The authors declare that the research was conducted in the absence of any commercial or financial relationships that could be construed as a potential conflict of interest.

Copyright (c) 2017 Ab Mutalib, Md Yusof, Abdul and Jamal. This is an open-access article distributed under the terms of the Creative Commons Attribution License (CC BY). The use, distribution or reproduction in other forums is permitted, provided the original author(s) or licensor are credited and that the original publication in this journal is cited, in accordance with accepted academic practice. No use, distribution or reproduction is permitted which does not comply with these terms. 